\title{
Proximal femoral focal deficiency of the fetus - early 3D/4D prenatal ultrasound diagnosis.
}

\author{
Marek J Kudla', Aleksandra Beczkowska-Kielek², Katarzyna Kutta², Justyna Partyka-Lasota ${ }^{3}$
}

${ }^{1}$ Clinical Department of Oncological Gynecology, Obstetric and Gynecology, Chair of Women's Health, Medical University of Silesia, ${ }^{2}$ Medical University of Silesia, ${ }^{3}$ Department of Gynecology and Obstetrics, Medical University of Silesia, Katowice, Poland

\begin{abstract}
Proximal Femoral Focal Deficiency (PFFD) is a rare congenital syndrome of unknown etiology. Additional disorders can be present up to $70 \%$ of PFFD cases. Management (including termination) depends on the severity of the malformation. We present a case of a 32-year-old woman referred for routine ultrasound examination in the $12^{\text {th }}$ week of pregnancy. Detailed 3D/4D evaluation revealed asymmetry of lower limbs and diagnosis of isolated PFFD was established. Parents were fully informed and decided to continue the pregnancy. We stress here the importance of early 3D/4D ultrasound diagnosis. Our paper presents the earliest case where the diagnosis of PFFD was established with 3D/4D ultrasound.

Keywords: prenatal diagnosis, fetal development, prenatal ultrasonography, 3D/4D ultrasonography
\end{abstract}

\section{Introduction}

Proximal Femoral Focal Deficiency (PFFD) is a rare congenital syndrome occurring in 1 out of 50000 200000 individuals [1]. It is a complex and challenging problem - due to the impaired development of the proximal part of the femur in the trochanteric region, the entire limb is shortened. Also biomechanical disorders (i.e. impaired rotation) are observed. There are also some other long bone malformations associated with PFFD, the most common being fibular hypoplasia (up to $70 \%$ cases). Although there are no chromosomal aberrations observed [2]. Management (including pregnancy termination) depends on the severity of the malformation.

Received 01.02.2016 Accepted 22.02.2016

Med Ultrason

2016, Vol. 18, No 3, 397-399

Corresponding author: Marek J Kudla MD PhD

Clinical Department of Oncological

Gynecology, Obstetric and Gynecology,

Chair of Women's Health,

Medical University of Silesia, Poland

65 Panewnicka street, 40-760 Katowice, Poland

Phone: +48 501377811

E-mail: marekkudla@hotmail.com

\section{Case report}

A 32 year old patient (gravida 3, para 2) was referred in the $12^{\text {th }}$ week of pregnancy due to the significant asymmetry of fetal lower limbs detected in an obstetric US examination. No family history or risk factors were present. Detailed ultrasound in the $12^{\text {th }}$ week revealed asymmetry of lower limbs - left femur length (FL) $15.4 \mathrm{~mm}$ while right only $4.6 \mathrm{~mm}$. Difference in the limbs length was displayed using trans-vaginal $2 \mathrm{D}, 3 \mathrm{D}$ and $4 \mathrm{D}$ projection (fig 1a-b). Parents were fully informed right after this examination and decided to continue the pregnancy. Follow up examination ( $24^{\text {th }}$ week) confirmed asymmetry of left and right femoral bones ( $45 \mathrm{~mm}$ and $17 \mathrm{~mm}$ respectively) (fig 1c). Mobility of the right limb was limited to the lower leg. Other parts of the fetus body (such as pelvis, thorax and skull) were without any abnormalities and biometric parameters such as biparietal diameter (BPD), head circumference (HC) and abdominal circumference (AC) were within normal range of values. No abnormalities were present in the right, nor in the left foot. PFFD was diagnosed and followed by a series of ultrasound examinations, which did not reveal any other skeletal malformations. Delivery by Cesarean Section was performed 


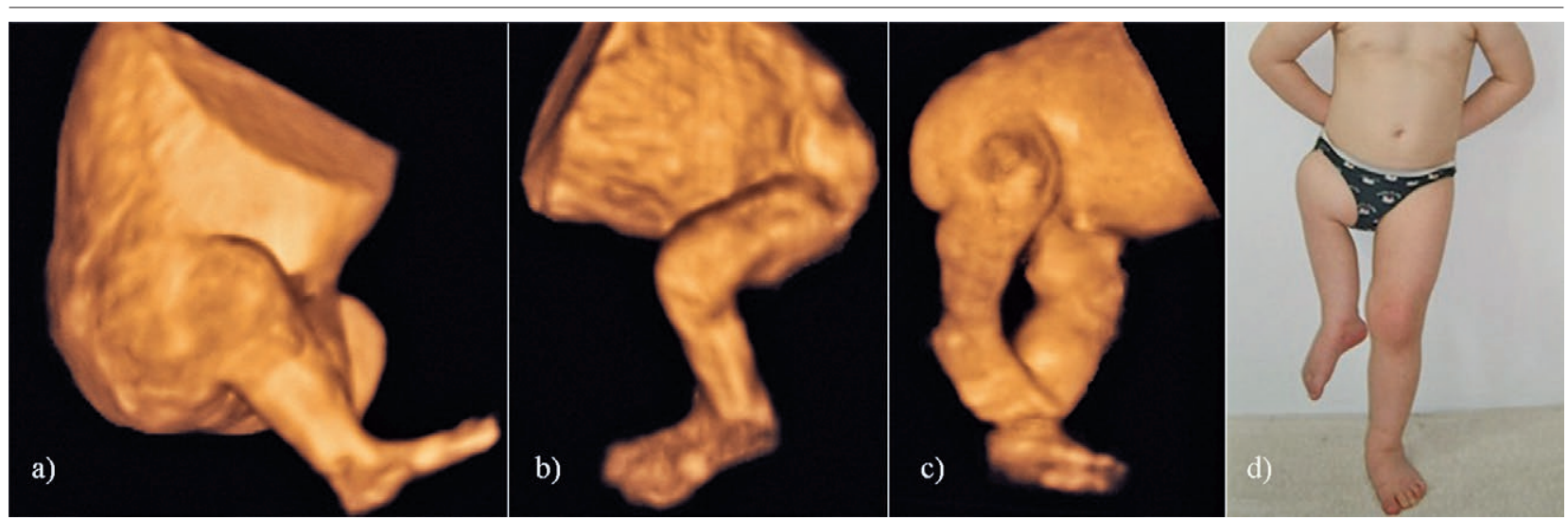

Fig 1. a) 3D render image of right lower extremity presenting evident shortened thigh in 12 th week of pregnancy; b) $3 \mathrm{D}$ render image of left lower extremity in 12th week of pregnancy; c) 3D render reconstruction of the lower extremities in 24th week of pregnancy. Difference in lower limbs length is visualized; d) Picture of the 3 year old patient - beside o the PFFD no other dysmorphic features (including facial and other long bones malformations) were present.

in the $38^{\text {th }}$ week of pregnancy and a baby girl $(2970 \mathrm{~g} /$ $53 \mathrm{~cm}$ ) was born with an Apgar score of 9. Diagnosis of isolated PFFD was confirmed. The child is 3 years old now (fig 1d), developing properly and presents normal activity by using a right extremity prosthesis.

\section{Discussions}

PFFD occurs in $0.5-2$ out of every 100000 births, mostly $(85-90 \%)$ as a unilateral type. [3] This is a rare congenital deformation of the trochanteric part of the femur, resulting in varying degrees of limb shortening. It can affect the head of the femur or/and acetabulum as well, causing article dysfunction, restricting movements and internal rotation of the limb [4] Etiology of this syndrome is not clearly known. Factors associated with improper embryonic bone development between the 4th and 6th weeks of pregnancy such as: hyperglycemia, medications (i.e. thalidomide), viral infection, ischemia or trauma are suspected. No genetic patterns associated with PFFD were detected, although family cases of this syndrome were reported. On the other hand there are more complex syndromes where femur hypoplasia is also observed and genetic mutations are found i.e. Fuhrmann syndrome (defects in WNT7A gene) causing multiple malformations of long bones, thorax and genitourianary anomalies.

Manifestation of PFFD depends on the grade of the femoral bone hypoplasia and requires an individual approach. There can be additional developmental disorders in up to $70 \%$ of PFFD cases, including fibular hemimeilia (most common), congenital talipes equinovarus (CTEV), oligodactyly, tibia bone binding, absence of knee cruciate ligaments or patella, and spinal deformity. [2].
That is why the detection of a short femur should be followed by detailed visualization combined with an extended differential diagnosis to exclude other defects. Differential diagnosis of the PFFD consists of: Femur/ Fibula/Ulna Complex (FFU) - confirmation of shortening of the femur, fibula and tibia; Femoral Dysplasia/ Unusual Facies Syndrome (FH/UFS) - confirmation of bilateral femoral dysplasia, micrognathia, palatoschisis, nose shortening, medial cleft lengthened, thin upper lip, upper limbs movement restriction, and rarely genitourinary congenital disorders; and Al-Awadi/Raas-Rothschild Syndrome known also as Limb/Pelvis-Hypoplasia/ Aplasia Syndrome (LPHA) - confirmation of complex upper and lower limbs malformations

Summarizing, in order to diagnose PFFD, particularly anomalies of the fetus's face and long bones need to be excluded. Detailed visualization obtained thanks to 3D and 4D options helps to eliminate complex congenital syndromes from differential diagnosis.

To the best of our knowledge the case reported herein is the earliest diagnosed PFFD syndrome detected with $3 \mathrm{D} / 4 \mathrm{D}$ ultrasound, although we have to stress that $2 \mathrm{D}$ diagnosis of femur hypoplasia in first trimester (among other fetal problems ) had already been reported in 1992 by Bronstein and Deutsch [5].

There are some other papers reporting PFFD detected later during pregnancy (second trimester) with no follow up as most of those pregnancies were terminated $[6,7,8]$.

Establishing early prenatal diagnosis of PFFD (especially its isolated form) helps to choose best management strategy. 3D and 4D options enable explaining the nature of the syndrome to parents as early as in the first trimester. It also helps to plan future rehabilitation as well as to select a proper way of prosthesising. 


\section{References}

1. Oppenheim WL, Setoguchi Y, Fowler E. Overview and comparison of Syme's amputation and knee fusion with the van Nes rotationplasty procedure in proximal femoral focal deficiency. In: Herring JA, Birch J, eds. The Child With a Limb Deficiency. Chicago, Ill: American Academy of Orthopaedic Surgeons; 1998.

2. Ellul M, Chircop M, Grixti C, Grech V. Proximal femoral focal deficiency - a case report. Malta Med J 2008; 20: 42-43.

3. Camera G, Dodero D, Parodi M, Zucchinetti P, Camera A. Antenatal ultrasonographic diagnosis of a proximal femoral focal deficiency. J Clin Ultrasound 1993; 21: 475-479.

4. Anton CG, Applegate KE, Kuivila TE, Wilkes DC. Proximal femoral focal deficienty (PFFD): more that an abnormal hip. Semin Musculoskelet Radiol 1999; 3: 215-226.
5. Bronstein M, Deutsch M. Early diagnosis of proximal femoral deficiency. Gynecol Obstet Invest 1992; 34: 246-248.

6. Mailath-Pokorny M, Timor-Tritsch IE, Monteagudo A, Mittal K, Konno F, Santos R. Prenatal diagnosis of unilateral proximal femoral focal deficiency at 19 weeks' gestation: case report and review of the literature. Ultrasound Obstet Gynecol 2011; 38: 594-597.

7. Lin TH, Chung CH, Shih JC, Lin CH, Lee CN, Su YN. Prenatal diagnosis of proximal femoral focal deficiency: A case report and literature review. Taiwan J Obstet Gynecol 2013; 52: 267-269.

8. La Torre R, Mastrone M, Coacci F, et al. Severe hypoplasia of the right femur, ipsilateral agenesia of the fibula and twisted right foot in a 24-week-old fetus with proximal femoral focal deficiency (PFFD). Clin Exp Obstet Gynecol 2003; 30: 65-66. 